\title{
Design and implementation of a smart antenna system for UHF vehicle-mounted communication
}

\author{
Lin Zhou, Huotao Gao ${ }^{\text {a) }}$, Huajun Zhang, Hao Li, \\ Huaqiao Zhao, and Fan Wang \\ Electronic Information Department, Wuhan University, \\ Luoyu Road No.129, Wuhan, 430072, China \\ a)gaoght863@163.com
}

\begin{abstract}
In this paper, the design and implementation of a smart antenna system for ultra high frequency (UHF) vehicle-mounted communication is proposed. The project adopts the improved multiple signal classification (MUSIC) algorithm which realized in field programmable gate array (FPGA), and implements robust superdirective beamforming algorithm based on digital signal processor (DSP). They contribute to achieving super-resolution target direction-finding and gaining output beam signals. This solution has greatly improved the signal interference noise ratio (SINR) and the signal's intensity in communication. The experimental results indicate that the system works smoothly with a rapid response and a strong stability, and satisfies the demand of engineering application.
\end{abstract}

Keywords: vehicle-mounted communication, smart antenna, directionfinding, digital beam forming, interference suppression

Classification: Electron devices, circuits, and systems

\section{References}

[1] S. A. Schelkunoff: Bell Syst. Tech. J. 22 (1943) 80. DOI:10.1002/j.1538-7305. 1943.tb01306.x

[2] A. M. Zhang and X. Q. Wang: Appl. Electron. Tech. 38 [1] (2012) 94.

[3] R. O. Schmidt: IEEE Trans. Antennas Propag. 34 (1986) 276. DOI:10.1109/ TAP. 1986.1143830

[4] L. Vachhani, K. Sridharan and P. K. Meher: IEEE Trans. Circuits Syst. II, Exp. Briefs 56 (2009) 61. DOI:10.1109/TCSII.2008.2010169

[5] H. J. Zhang, H. T. Gao, Q. C. Zhou, L. Zhou and F. Wang: IEICE Electron. Express 11 (2014) 20130919. DOI:10.1587/elex.11.20130919

[6] D. F. Xu, J. Cheng and C. H. Zhang: Command Inf. Syst. Technol. 2 [2] (2011) 10.

[7] B. Friedlander and A. J. Weiss: Acoustics, Speech, and Signal Processing, 1988. ICASSP-88 (1988) 2681. DOI:10.1109/ICASSP.1988.197201

[8] Z. G. Ma, L. P. Zhao, Z. X. Li and A. W. Li: Microcomputer \& Its Applications [10] (2010) 24.

[9] D. H. Chen: Ph.D thesis University of Electronic Science and Technology, Chengtu, China (2008). 


\section{Introduction}

Smart antenna system is based on the principle of adaptive antenna array, using antenna array beam forming technology to make the main lobe adjusted to the desired signal [1]. In the UHF vehicle-mounted communication system, the smart antenna technology can effectively suppress multi-path interference, co-channel interference, multiple access interference, and other various interferences [2]. However, gain-phase errors are inevitable in engineering application. The traditional active calibration method and joint iteration method get a poor estimation on the moving platform, which causes a performance deterioration and even failure of MUSIC [3]. Considering this problem, we propose the improved MUSIC algorithm to simultaneously estimate the direction of arrival (DOA) and gain-phase errors without joint iteration. Theoretical analysis shows that it performs independently of phase errors and thus behaves well regardless of phase errors. This work obtains the characteristics of high estimation accuracy and quasi real-time. To some extend, this system can greatly improve signal transmission quality, use the spectrum effectively and increase the system capacity. All of them contribute to improving the communication quality in engineering application.

This paper will illustrate the design and implementation of the smart antenna system mentioned above. The system implement the improved algorithm based on FPGA. Through the efficient filtering and parallel design of the coordinate rotation digital computing (CORDIC) [4], the system greatly reduces the computation complexity. More importantly, the system realizes multi-objective distinguish and high accuracy direction-finding with a rapid response. Besides this, it implements a digital beamformer (DBF) [5] to obtain the output beam adjusted to the desired target. The final output communication signal both has higher gain and higher SINR. Therefore, it will enhance the anti-jamming intensity [6].

In the following, the algorithm theory is presented in section 2. Section 3 gives the detail design and implementation of this system. Closed-loop test and field experiment are shown in section 4. Finally, we come to a conclusion in section 5.

\section{The algorithm theory}

\subsection{Improved MUSIC algorithm for DOA and gain-phase errors}

Consider a planar array with $N$ omni-directional sensors and $K$ narrow-band farfield signals $s(t)$. The gain and phase errors are denoted as $G=\operatorname{diag}\left(\left[1, \rho_{2}, \cdots\right.\right.$, $\left.\left.\rho_{N}\right]^{T}\right)$ and $\Phi=\operatorname{diag}\left(\left[1, e^{j \varphi_{2}}, \cdots, e^{j \varphi_{N}}\right]^{T}\right)$, respectively. In this paper, $\operatorname{diag}\{\bullet\}$ defines a diagonal matrix. The array output vector is described as:

$$
x(t)=G \Phi A(\theta) s(t)+n(t),
$$

Where $A(\theta)=\left[a\left(\theta_{1}\right), a\left(\theta_{2}\right), \cdots, a\left(\theta_{K}\right)\right]$, with $a\left(\theta_{i}\right)$ is the ideal steering vector of array, and $n(t)$ represents the vector of additive noise. Calculating the covariance matrix of $x(t)$ as follows:

$$
R_{0}=E\left[x(t) x^{H}(t)\right]=G \Phi A R_{s} A^{H} \Phi^{*} G+\delta_{n}^{2} I_{N},
$$


Decomposing $R_{0}$, we have $R_{0}=\sum_{i=1}^{K} \lambda_{i} u_{i} u_{i}^{H}$, with $\lambda_{1} \geq \lambda_{2} \geq \cdots \geq \lambda_{K} \geq \lambda_{K+1}=$ $\cdots=\lambda_{N}=\delta_{n}^{2}>0$. Thus the estimation of $\delta_{n}^{2}$ can be given as:

$$
\hat{\delta}_{n}^{2}=\frac{1}{N-K} \sum_{i=K+1}^{N} \lambda_{i} .
$$

Define $R_{1}=R_{0}-\delta_{n}^{2} I_{N}=G \Phi A R_{s} A^{H} \Phi^{*} G$, and the $i$ th diagonal element of the matrix $R_{1}$ is denoted as $R_{1}(i, i)$. Gain errors can be estimated as:

$$
\hat{\rho}_{i}=\sqrt{\frac{R_{1}(i, i)}{R_{1}(1,1)}} \quad i=1,2, \cdots, N .
$$

Now we get the inverse matrix $G^{-1}=\operatorname{diag}\left(\left[1, \frac{1}{\rho_{2}}, \cdots, \frac{1}{\rho_{N}}\right]^{T}\right)$, then define $R_{2}=$ $G^{-1} R_{1} G^{-1}=\Phi A R_{S} A^{H} \Phi^{*}$, with the elements given by:

$$
\left[R_{2}\right]_{p, q}=e^{j\left(\varphi_{p}-\varphi_{q}\right)} \sum_{i=1}^{K}\left[a\left(\theta_{i}\right)\right]_{p}\left[a^{*}\left(\theta_{i}\right)\right]_{q} \delta_{i}^{2} .
$$

Here, we construct a covariance matrix $R_{3}=R_{2} \odot R_{2}^{T}$, where $\odot$ denotes dot product (element-wise multiplication), with the elements given by:

$$
\begin{aligned}
{\left[R_{3}\right]_{p, q} } & =\left[R_{3}\right]_{q, p}=\left[R_{2}\right]_{p, q}\left[R_{2}\right]_{q, p} \\
& =\sum_{i=1}^{K}\left[a\left(\theta_{i}\right)\right]_{p}\left[a^{*}\left(\theta_{i}\right)\right]_{q} \delta_{i}^{2} \sum_{j=1}^{K}\left[a\left(\theta_{j}\right)\right]_{q}\left[a^{*}\left(\theta_{j}\right)\right]_{p} \delta_{j}^{2} \\
& =\sum_{i=1}^{K} \sum_{j=1}^{K}\left\{\left[a\left(\theta_{i}\right)\right]_{p}\left[a^{*}\left(\theta_{j}\right)\right]_{p}\right\} \delta_{i}^{2} \delta_{j}^{2}\left\{\left[a\left(\theta_{i}\right)\right]_{q}\left[a^{*}\left(\theta_{j}\right)\right]_{q}\right\}^{*}
\end{aligned}
$$

Denote $b\left(\theta_{i}, \theta_{j}\right)=a\left(\theta_{i}\right) \odot a^{*}\left(\theta_{j}\right)$ as the new steering vectors related to $R_{3}$. It is clear that $b\left(\theta_{i}, \theta_{i}\right)=1_{N}$, Eq. (6) can be simplified as:

$$
\left[R_{3}\right]_{p, q}=\delta_{0}^{2}+\sum_{i=1}^{K} \sum_{\substack{j=1 \\ j \neq i}}^{K}\left[b\left(\theta_{i}, \theta_{j}\right)\right]_{p} \delta_{i}^{2} \delta_{j}^{2}\left[b^{*}\left(\theta_{i}, \theta_{j}\right)\right]_{q},
$$

Where $\delta_{0}^{2}=\sum_{l=1}^{K} \delta_{l}^{4}$, the new covariance matrix $R_{3}$ can be modified as:

$$
R_{3}=\sum_{i=1}^{K} \sum_{\substack{j=1 \\ j \neq i}}^{K} b\left(\theta_{i}, \theta_{j}\right) \delta_{i}^{2} \delta_{j}^{2} b^{H}\left(\theta_{i}, \theta_{j}\right)+\delta_{0}^{2} I_{N} .
$$

Based on signal subspace theory, we obtain:

$$
\operatorname{Col}\left(R_{3}\right)=\operatorname{span}\left\{1_{N}, b\left(\theta_{i}, \theta_{j}\right) \mid i \neq j, i, j=1,2, \cdots K\right\},
$$

By decomposing, we have $R_{3}=\sum_{i=1}^{N} \beta_{i} u_{i}^{\prime} u_{i}^{\prime H}$, where the eigenvalues are arranged in descending order. Denote $k_{1}=\operatorname{rank}\left(R_{3}\right)=K(K-1)+1$, then the noise subspace matrix can be presented as:

$$
U_{n}=\left[u_{k_{1}+1}^{\prime}, u_{k_{1}+2}^{\prime}, \cdots, u_{N}^{\prime}\right] .
$$

In consequence, the improved DOA estimation method is proposed using the methodology of the conventional MUSIC method. The two-dimensional spatial spectrum is defined as follows: 


$$
P\left(\theta_{i}, \theta_{j}\right)=\frac{1}{\left\|U_{n}^{H} b\left(\theta_{i}, \theta_{j}\right)\right\|_{2}^{2}},
$$

As $\theta_{i}$ and $\theta_{j}$ both represent the DOA, we give a dimension spatial spectrum to reduce the peak searching complexity which presented as:

$$
\begin{aligned}
& P_{\text {music }}(\theta)=f(\theta) \\
& f(\alpha)=\max \left[\left.P\left(\alpha, \theta_{j}\right)\right|_{\theta_{j}<\alpha}\right]
\end{aligned}
$$

By searching the peaks of $P_{\text {music }}(\theta)$, we get the DOA estimation, which behaves well regardless of phase errors based on Eq. (6). Obviously, the improved algorithm achieves super-resolution target direction-finding.

Finally, we get the estimation of phase errors using the method in [7]. Denote $\eta=\left[\varphi_{1}, \varphi_{2}, \cdots, \varphi_{N}\right]^{T}$, and $\operatorname{angle}(\bullet)$ represents the phase of a complex number. The detailed estimation is described as follows:

$$
\eta=\operatorname{angle}(z)
$$

Where:

$$
\begin{aligned}
z & =\frac{\Omega^{-1} w}{w^{T} \Omega^{-1} w} \\
\Omega & =\sum_{i=1}^{K} D^{H}\left(\theta_{i}\right) U_{n} U_{n}^{H} D\left(\theta_{i}\right) \\
D\left(\theta_{i}\right) & =\operatorname{diag}\left[a\left(\theta_{i}\right)\right] \\
U_{n} & =\left[u_{K+1}, u_{K+2}, \cdots, u_{N}\right] \\
w & =[\underbrace{1,0, \cdots, 0}_{N}]^{T}
\end{aligned}
$$

\subsection{The implementation of beamforming}

According to the results of DOA, we implement the robust adaptive beamforming based on null widening. Then we send the weight coefficient to DBF module which realized in FPGA.

As $\overline{\theta_{k}}$ is the incidence angle of the interference, and we substitute $v_{k}$ for $\pi \sin \overline{\theta_{k}}$. Thus, the average spatial correlation matrix is given as follows:

$$
\bar{R}=\sum_{k=1}^{K} \delta_{k} \int f\left(v_{k}\right) a\left(\theta_{k}\right) a^{H}\left(\theta_{k}\right) d v_{k}+\delta_{n}^{2} I,
$$

In above formula, $f\left(v_{k}\right)$ and $a\left(\theta_{k}\right)$ are the probability density function of $v_{k}$ and the steering vector of adaptive array antenna respectively, and $\delta_{k}$ represents the signal's power. Here, we define that $\delta_{p}^{2}=\frac{1}{K} \sum_{k=1}^{K} \delta_{k}^{2}$, and the $(m, n)$ 's element of $\bar{R}$ can be expressed below:

$$
[\bar{R}]_{m, n}=\sum_{k=1}^{K} \delta_{k} \times \exp \left[-j(m-n) \pi \sin \left(\theta_{k}\right)\right] \times \exp \left[-\frac{1}{2} \delta_{p}^{2}(n-m)^{2}\right]+\delta_{n}^{2} I,
$$

Therefore, Eq. (15) can be rewritten as: 


$$
\bar{R}=\sum_{k=1}^{K} \delta_{k} a\left(\theta_{k}\right) a^{H}\left(\theta_{k}\right) \circ T\left(\theta_{k}, \delta_{p}^{2}\right)+\delta_{n}^{2} I,
$$

Here, matrix $T\left(\theta_{k}, \delta_{p}^{2}\right)$ can be presented as:

$$
\left[T\left(\theta_{k}, \delta_{p}^{2}\right)\right]_{m, n}=\exp \left\{-\frac{1}{2} \delta_{p}^{2}\left[(m-n) \pi \cos \left(\theta_{k}\right) / 180\right]^{2}\right\},
$$

The effect of matrix is widened the incident angle for disturbance signal. Thus, the system can obtain a pattern with null widening in the direction of interference.

\section{Design and implementation of the system}

The detail implementation of system block diagram is given in Fig. 1(a). In this design, we realize peripheral interface configuration and parallel computing in FPGA chip, and adopt DSP chips [8] to implement the signal processing algorithm. In the final stage, the communication between this system and upper computer is achieved via the transport protocol of universal asynchronous receiver transmitter (UART).

\subsection{Digital down conversion (DDC) module}

Antenna signal is high-speed sampled using the chips AD924x after passing through amplifiers and filters. The sampling rate is $40 \mathrm{MHz}$, while the signal bandwidth is only $300 \mathrm{KHz}$. In order to improve the follow-up data processing efficiency and reduce the time overhead, we implement the digital orthogonal transformation in FPGA to achieve the DDC function.

In this design, numerically controlled oscillator (NCO) is realized with the look-up table method based on the principle of direct digital synthesizer (DDS). Due to the difficulty of designing a high order single-stage filter for hardware, it adopts 3-stage filters: cascaded integrator comb filter (CIC), half band filter (HB) and finite impulse response filter (FIR) [9], the decimation factors of that are respectively A, B (single-stage is 2) and $C$, and the total decimation factor is $\mathrm{A} \times \mathrm{B} \times \mathrm{C}$. The block diagram of digital quadrature demodulation is shown in Fig. 1(b).

\subsection{Up-conversion module}

As this system works at UHF, it is difficult to obtain the direct digital up frequency conversion with a chip. What's more, a large amount of spurious signal will be generated in this process. Both of them will bring a bad influence to the quality of radio communication. Therefore, we generate a fixed carrier frequency output signal which carrying all the information, which then goes through the intermediate frequency narrow-band filter, mixer and numerical control frequency modulation (NC FM) filter in turn. After these process mentioned above, the final radiofrequency (RF) signal wins the characteristics of high gain and large signal noise ratio (SNR). The implementation of up-conversion is shown in Fig. 1(c). 


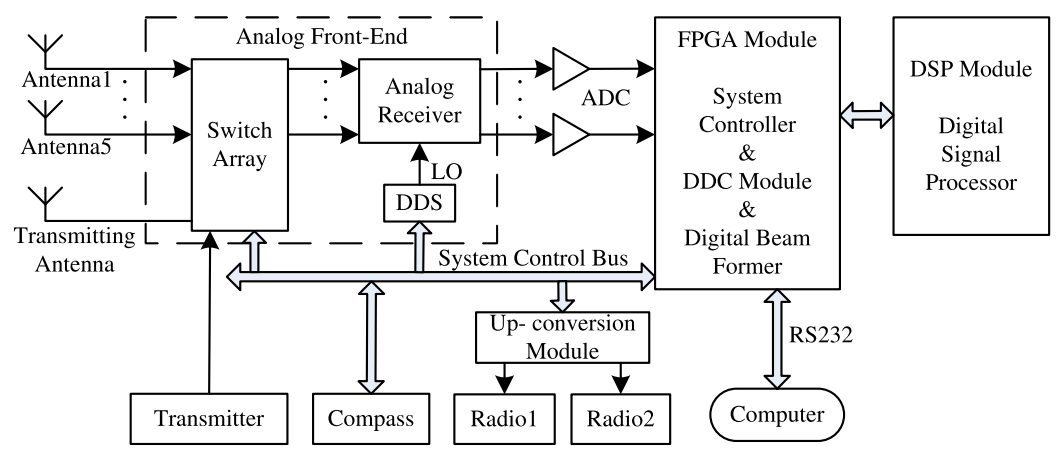

(a)

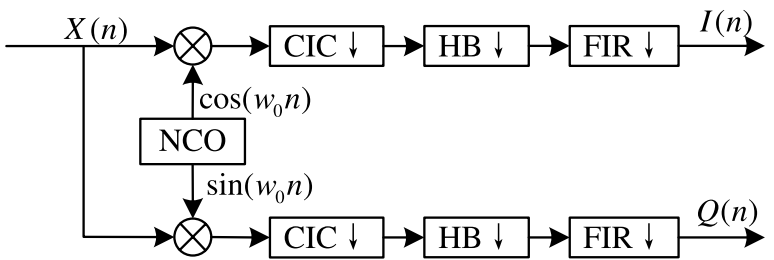

(b)

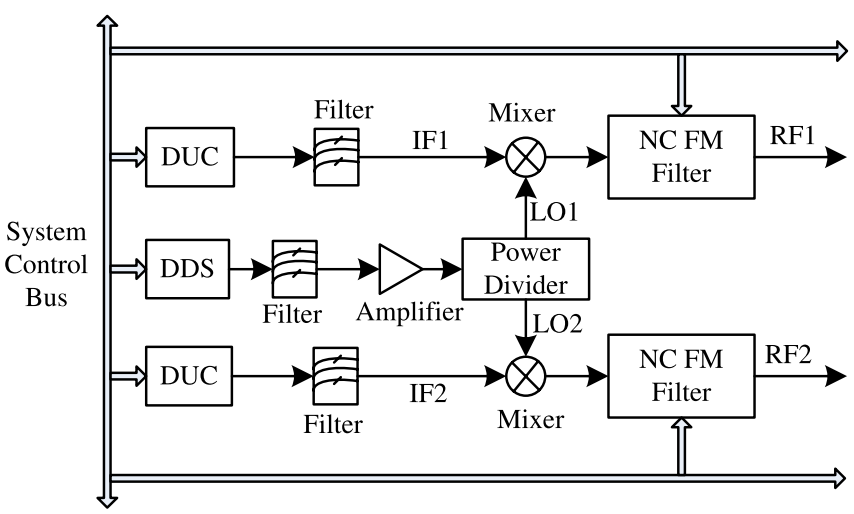

(c)

Fig. 1. (a) System block diagram. (b) Block diagram of DDC module. (c) Block diagram of up-conversion module.

\section{Test results}

\subsection{Closed-loop test}

In this closed-loop test, the system works at $400.2 \mathrm{MHz}$, where the input source power with $-90 \mathrm{dBm}$. Through a delay cables, we get the simulated DOA as $36.0^{\circ}$.

Fig. 2(a) and Fig. 2(b) have shown the channel consistency after finishing the channel calibration, the amplitude and phase errors are less than $0.03 \mathrm{~dB}$ and $0.3^{\circ}$, respectively. Comparing the output power of Fig. 2(c) and Fig. 2(d), it is obvious that the communication signal going through the smart antenna system has been enhanced more than $13 \mathrm{~dB}$, this will greatly improve the communication quality in application. The Table I shows the response time of target direction-finding and distinguish in this system, where $n$ denotes the sampling number in covariance matrix calculation. 


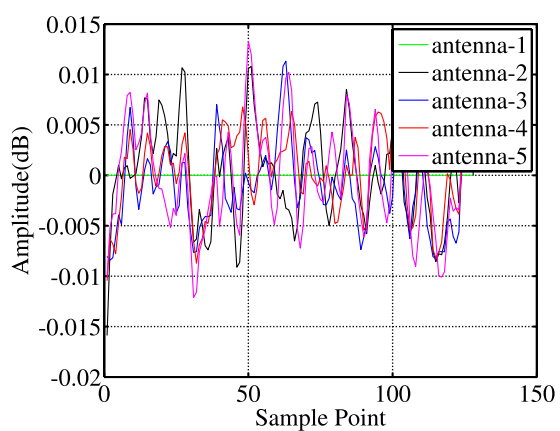

(a)

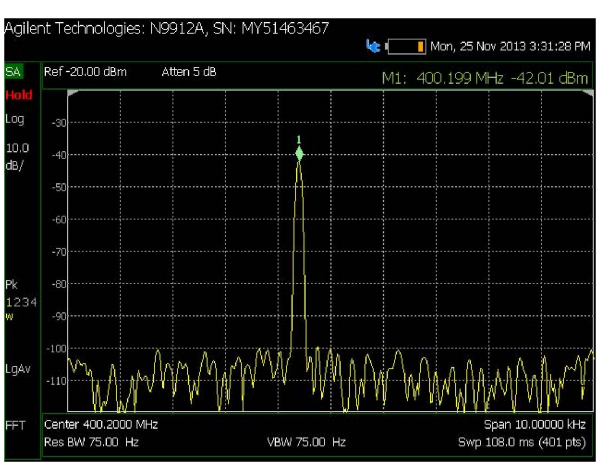

(c)

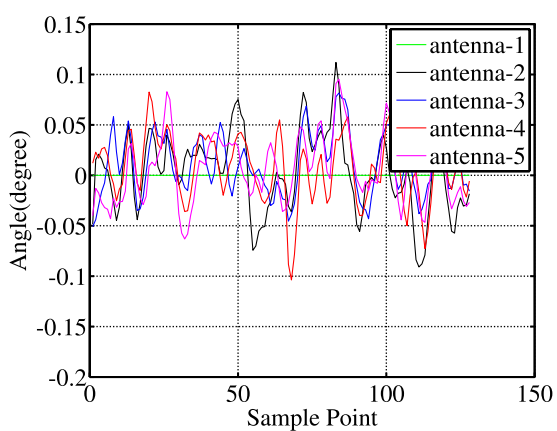

(b)

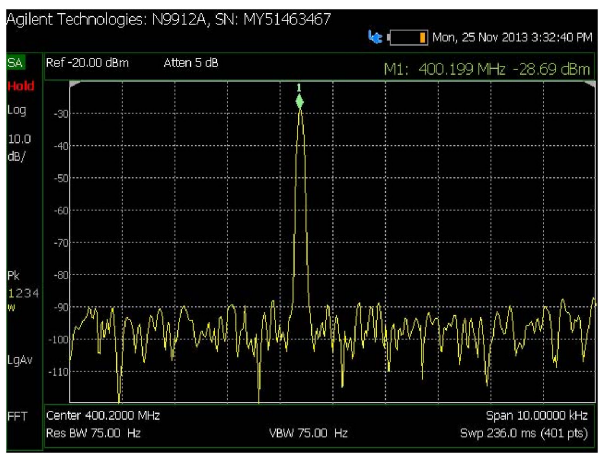

(d)

Fig. 2. (a) Amplitude consistency. (b) Phase consistency. (c) Single antenna output. (d) Smart antenna output.

Table I. Response time.

\begin{tabular}{|c|c|c|c|c|c|c|c|c|}
\hline Snapshots $/ \mathrm{n}$ & 512 & 256 & 128 & 64 & 32 & 16 & 8 & 4 \\
\hline DOA $\left(^{\circ}\right)$ & 36.0 & 36.0 & 36.0 & 36.0 & 36.0 & 35.9 & 35.9 & 35.8 \\
\hline Time $(\mathrm{ms})$ & 1.88 & 1.62 & 1.49 & 1.38 & 1.35 & 1.33 & 1.32 & 1.31 \\
\hline
\end{tabular}

\subsection{Field experiment in Wuhan}

This experiment was done in Wuhan on December 2013. We adopt a uniform circular array with five units, and the direction-finding error is acceptable within $7^{\circ}$ in theory design, where the DOA of testing targets are $68^{\circ}$ and $345^{\circ}$, respectively. The experiment platform is shown in Fig. 3(a). By monitoring the external environment, we choose the working frequency: $331.1 \mathrm{MHz}$.

The Fig. 3(b) shows the result with targets both working at $331.1 \mathrm{MHz}$. Quite on the contrary, the result of the different frequencies testing is given in Fig. 3(c), where the targets working at $331.1 \mathrm{MHz}$ and $331.05 \mathrm{MHz}$, respectively. The experiment proves that the system has realized multi-objective distinguish in both conditions with the direction finding error is less than $5^{\circ}$.

We can't receive any information from the radio station using the original antenna, as the two signals are mixed totally in such testing conditions. However, the smart antenna system can produce an adaptive beam, with the main beam pointing towards the desired target and forming null point in the direction of interferences. The corresponding beam patterns are shown in Fig. 3(d), and SINR is greater than $20 \mathrm{~dB}$. Therefore we can restore the original speech signal, and make the normal communication with each other come true. 


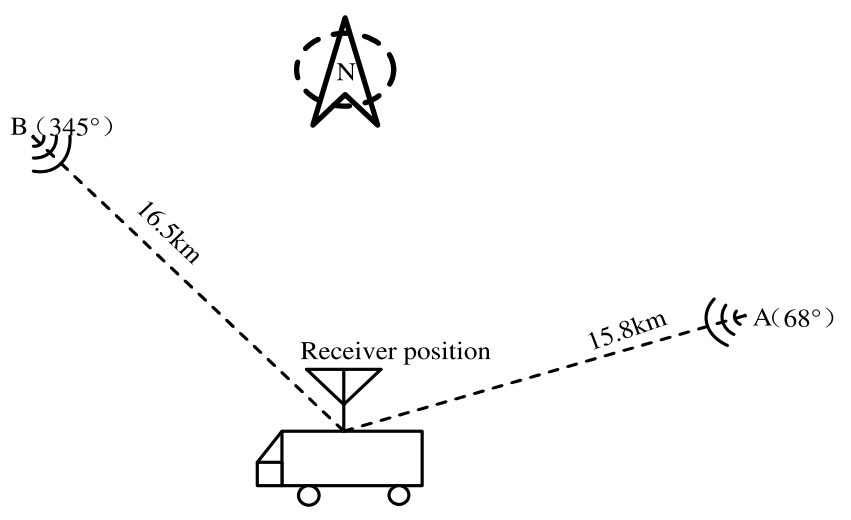

(a)

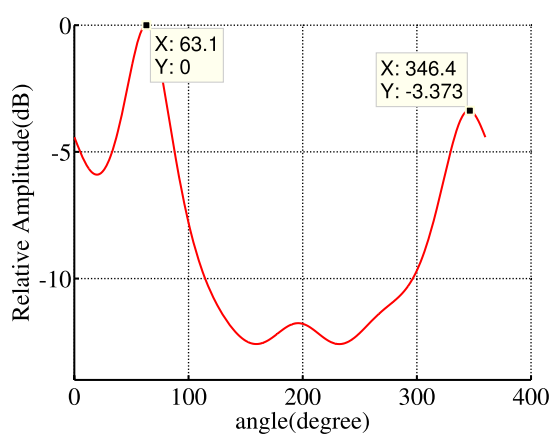

(b)

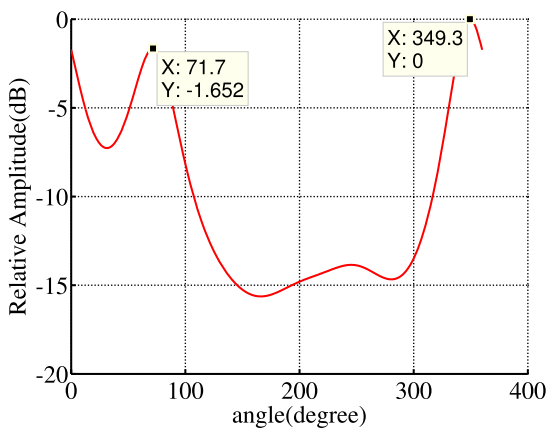

(c)

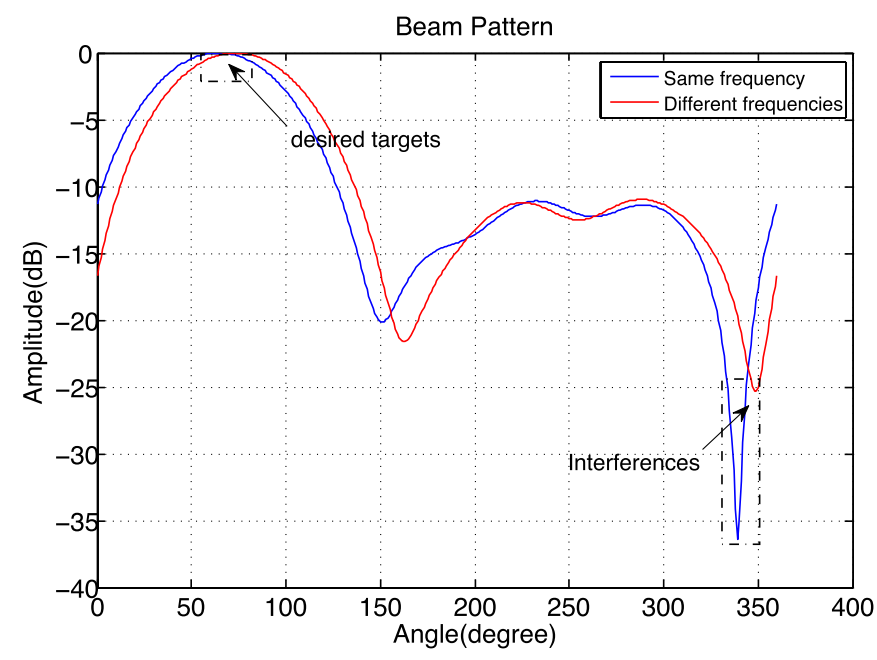

(d)

Fig. 3. (a) The experiment platform. (b) DOA of same frequency testing. (c) DOA of different frequencies testing. (d) The beam patterns

\section{Conclusion}

The design and implementation of a smart antenna system for UHF vehiclemounted communication has been illustrated in this paper. The program makes the most of the capability of FPGA's parallel processing to obtain a large capacity of data processing and a rapid response of DOA. Besides the implementation of beamforming algorithm, it also gets a flexible system parameters' configuration based on multiple DSP chips. According to the illustrated closed-loop test and field 
experiment results, this project not only achieves multiple target direction-finding, but also wins a high gain performance and SINR in vehicle-mounted communication. The smart antenna system can work steadily under the complex environment, and is feasible and upgradable in engineering application.

\section{Acknowledgments}

This work is supported by the Fundamental Research Fund for the Central Universities under grant 201221220213 and T201221208. The authors wish to express their gratitude to the editor and the anonymous reviewers. 